\title{
Pyramid of Ferrein
}

National Cancer Institute

\section{Source}

National Cancer Institute. Pyramid of Ferrein. NCI Thesaurus. Code C33435.

Conical structures of the cortical portion of the kidney. The apices of these structures reach the periphery of the kidney, and the bases are applied to the medullary portion. 